\title{
Wireless Erasure Networks With Feedback
}

\author{
Brian Smith \\ Department of ECE \\ University of Texas at Austin \\ Austin, TX 78712 \\ bsmith@ece.utexas.edu
}

\author{
Babak Hassibi \\ Department of Electrical Engineering \\ California Institute of Technology \\ Pasadena, CA 91125 \\ hassibi@systems.caltech.edu
}

\begin{abstract}
Consider a lossy packet network of queues, communicating over a wireless medium. This paper presents a throughput-optimal transmission strategy for a unicast network when feedback is available, which has the following advantages: It requires a very limited form of acknowledgment feedback. It is completely distributed, and independent of the network topology. Finally, communication at the information theoretic cut-set rate requires no network coding and no rateless coding on the packets. This simple strategy consists of each node randomly choosing a packet from its buffer to transmit at each opportunity. However, the packet is only deleted from a node's buffer once it has been successfully received by the final destination.
\end{abstract}

\section{INTRODUCTION}

It is well known that in the memoryless point-to-point channel model, feedback can never increase the value of the information theoretic capacity [1]. However, there are several significant advantages to having feedback. Feedback allows coding strategies which can significantly increase the probability of error decay, for example the Schalkwijk-Kailath scheme for additive Gaussian noise channels [5]. Feedback can also allow transmission strategies with extremely simple coding algorithms. Specifically, consider the binary symmetric erasure channel. When feedback is available, the transmitter can simply repeat each bit until successfully received. Capacity is achieved, and in some sense, no coding whatsoever is required.

In this paper, a unicast model of a lossy wireless network of queues is considered, similar in spirit to the wireless erasure network [3]. Our network model is characterized by independent erasure channels/loss probabilities on a directed graph, a wireless broadcast requirement, asynchronous transmission timing, and a single source-destination pair. With transmit opportunities occurring as a unit rate Poisson process, a transmission by one node will be received independently with some fixed probability by each other node in the network. The network model will allow general feedback, but it will be shown that only a very limited form of acknowledgment feedback is required to achieve the throughput-optimal cutset capacity. The primary differences between our model and that of [3] are first, the availability of feedback, and second,

The first author would like to acknowledge funding from an ARO Young Investigator Award. The work of the second author was in parts supported by the National Science Foundation under grant CCF 0729203, by the David and Lucille Packard Foundation and by Caltech's Lee Center for Advanced Networking. an asynchronous, memoryless arrival process (rather than a slotted-time model).

A similar asynchronous network model was studied in [2]. The authors' model demonstrates the usefulness of network coding: with no feedback, but allowing network coding and additionally, a packet header describing the linear combinations of data packets included in the transmission, they demonstrate the achievability of the cut-set bound. Our work highlights somewhat of a dual statement: without any sort of coding, but with feedback, the same cut-set packet rate is achievable.

The paper [4] also is concerned with a similar wireless lossy packet network model. With a backpressure algorithm, throughput-optimality in a multi-commodity sense is also achieved in a multiple-source multiple-destination network. This algorithm requires link-level feedback, and for each node to maintain knowledge of the queue state of, in worst case, every other node in the network. It provides a decision process, when multiple nodes in the network receive copies of the same packet, to decide which (if any) of those nodes should keep that packet and attempt to forward it onward.

In contrast, the routing algorithm described in this paper is completely decentralized and requires no conferencing among nodes to decide who should "keep" a packet that it has received. Instead, there will in general be multiple copies of each packet throughout the network.

Specifically, the algorithm is as follows: Whenever a node has an opportunity to transmit a packet, it will randomly choose one packet from its buffer. Every time that a packet successfully reaches the final destination node, that node will (errorlessly) broadcast an acknowledgment to every node in the system stating that this particular packet has successfully completed its transit of the network. Only after receiving this acknowledgment from the final destination node will any node remove the packet from its buffer. Indeed, the entire network will then flush that packet from all the buffers. This paper shows via Foster's Theorem and an application of an appropriate and novel Lyapunov function the stability of all network queues under this operation as long as the input data rate is less than the min-cut of the network. The authors are unaware of previous uses of an exponential Lyapunov function of the form we consider in showing stability results.

The advantages of this throughput optimal strategy include

- It requires no coding, particularly no network coding at intermediate nodes. 
- The only information that a packet header must contain is an identifier - no additional information is required.

- It is completely decentralized. No coordination or conferencing, other than the acknowledgment feedback, is required.

- It is topology independent. No node other than the source needs any information about the layout of the network. The source must only be given the value of the min-cut, which could even be adaptively estimated, if desired.

- The only feedback required, a simple acknowledgment from the destination, is practically already implemented in real systems.

The main thrust of this paper: A demonstration that, in this lossy wireless network, feedback obviates the need for coding, network coding in particular.

\section{NeTwOrk MOdEL AND NotATION}

Consider a directed (possibly cyclic) graph $G(V, E)$ with $n+2$ nodes: a source node, a destination node, and $n$ intermediate nodes. Label the source node $s$, the destination $d$, and index the other nodes as $i \in 1, \ldots, n$. To each edge pair $(i, j) \in V \times V$ assign an erasure probability $0 \leq \epsilon_{i j} \leq 1$. If the directed edge $(i, j)$ does not exist in the graph, then assign $\epsilon_{i j}=1$. Define $\mu_{i j}=1-\epsilon_{i j}$.

Because of the wireless nature of the model, when a node $i$ transmits a packet, each other node in the system $j$ has the probability $\mu_{i j}$ of successfully receiving that packet. The events that packets are dropped are independent, that is i.i.d. across time for any fixed edge $(i, j)$, and independent between every pair of edges.

An infinite buffer exists at each node in the network. Packets will exogenously arrive at the source node $s$ according to a Poisson process with arrival rate $\lambda$. At average rate 1 exponentially distributed intervals, each node in the network (other than the destination node) receives an opportunity to transmit a packet.

Each packet has a unique identifier in its header. Therefore, if a node already has a copy of a particular packet and it receives that packet again, the contents of that node's buffer remain unchanged.

A feedback mechanism exists such that when the destination node receives a packet, it instantaneously, via a delay-free feedback, notifies all of the other nodes in the system of that fact. All nodes in the system then immediately remove that particular packet from their buffer. Finally, this asynchronous model does not consider any receiver interference or the possibility of simultaneous arrivals.

\section{CUt-SET Upper Bound AND Transmission STRATEGY}

Under any transmission strategy, the cut-set upper-bound remains valid. Intuitively, the cut-set upper-bound is obtained by dividing the network into two parts $S$ and $S^{C}$ and creating two super-nodes. That is, by allowing free, unlimited communication among the nodes in $S$ and among the nodes in $S^{C}$, we can only increase the capacity of the system.
With that in mind, let $S$ be a subset of the $n+2$ nodes such that $s \in S$ and $d \in S^{C}$. There are $2^{n}$ such subsets. Let $\mathcal{S}$ be the set of such subsets. The super-node created by joining all nodes in $S$ together will still have opportunities to transmit at exponentially distributed intervals, but now the sum rate will be $|S|$ - a rate of 1 for each node in $S$. For each node $i \in S$, because of the unlimited free communication on the right side of the cut in $S^{C}$, as long as one of the nodes $j \in S^{C}$ successfully receives the packet, we can count it in the total communication throughput. Therefore, define

$$
C(S)=\sum_{i \in S}\left(1-\prod_{j \in S^{C}} \epsilon_{i j}\right)
$$

as the cut-set capacity for the subset $S$, i.e. an upperbound on the rate of packets that can be transmitted across the $S-S^{C}$ cut, exactly as per [3]. The total throughput $T<C(S)$ then, for every subset $S$, and $R<\min _{S \in \mathcal{S}} C(S)$.

The authors would like to emphasize the key role that the subsets $S$ will play in the proof and the derivation of the stability results. The minimum of $C(S)$ over all $S-S^{C}$ cuts must emerge from any stability equations; therefore it is reasonable that each cut-set represented by $S$ must play a role. As will be further explained, the sets $S$ will become essential as indices to the variables $m_{S}$ which describe the state of our Markov chain model. It will become clear that as the state variable $m_{S}$ corresponding to the subset $S$ becomes large, the requirement $\lambda<C(S)$ becomes a dominant constraint.

The network operates in the following manner: At every transmission opportunity for a node, that node randomly chooses one of the packets in its buffer to transmit. If the buffer is empty, then that transmission opportunity is lost. Only when acknowledgment from the final destination $d$ is received will a node remove a packet from its buffer; therefore there are multiple copies of each packet in the network.

Theorem 1: Under this randomized transmission strategy, all queues in a wireless erasure network with feedback are stable as long as $\lambda<C(S)$ for all $S \in \mathcal{S}$.

At first glance, this randomized strategy seems unnecessarily wasteful. Consider a network which is a simple serial line of queues. In this case, it is obvious that an optimal strategy, when link-level feedback is available, is to stop attempting to transmit a packet (and remove it from one's queue) as soon as it is successfully received at the next queue down the line. Leaving a successfully transmitted packet in the queue could result in the retransmission of that packet, possibly wasting a transmission opportunity that could be put to better use sending a new packet.

However, the randomization is crucially important in achieving the minimum-cut value for this network and for a general network. To achieve the min-cut, it is essential that all transmitters on the min-cut boundary transmit packets at almost every channel use and that these packets be almost always distinct. As the input rate $\lambda$ increases, the min-cut slowly becomes the bottleneck of the network and the queues on its boundary will grow large. This will ensure that each 
transmitter always has a packet to transmit with high probability. The randomization in packet transmission guarantees that for such long queues the probability that two transmitters along the min-cut transmit the same packet is very low. Deterministic strategies, such as FIFO for example, cannot guarantee this without coordination, and so the randomized strategy is essential to achieving the optimal throughput in a completely decentralized manner.

\section{PRoOF PRELIMINARIES}

\section{A. Notation and Description of Markov Chain Model}

Before formally beginning the proof of Theorem 1, some additional notation must be defined.

The subset $S$ has already been defined to be an element of $\mathcal{S}$, which is essentially the power-set of $n$ nodes. Precisely, $\mathcal{S}$ differs from the power-set of $n$ only in that all $S \in \mathcal{S}$ always include the source node $s$ and never include destination node $d$. Equivalently, each subset $S$ can represent an index in the set $\left\{0,1,2, \ldots, 2^{n}-1\right\}$. With this notion, the length- $n$ binary expansion of $S$ indicates which of the $n$ nodes are contained within the subset $S$. This yields a one-to-one correspondence between subsets, cut-sets, and indices, all represented by the overloaded notation $S$.

A continuous time Markov chain model is used to describe the state of the queuing network. Transitions between states will occur when one of three different types of events happen in the network:

- A new packet is received by the source node $s$.

- A packet is successfully transmitted from some node $i$ in the system to some subset of the receivers.

- A packet is successfully received by the the destination node $d$ and therefore exits the network.

In the $n=1$ three node network, the size of the buffers at the source node $s$ and the intermediate node 1 are sufficient to describe any state of the system. Observe that by the given network operation protocol, no packet is deleted from a queue until it reaches the final destination, so that if a packet is present anywhere within the system, it must be present at the source node $s$. For a general network, however, the queue lengths do not provide a sufficient description of the network state, so we choose a different state definition.

Let $m_{1}$ be the number of packets which appear at both the nodes $s$ and 1 . Let $m_{0}$ be the number of packets which appear at the source node uniquely. Then the source node has a total of $m_{0}+m_{1}$ packets, while the relay node has exactly $m_{1}$ packets in its buffer. This state description can be generalized to an $n+2$ node network. The Markov chain describing the system state is a vector $\underline{m}$ with $2^{n}$ dimensions:

$$
\underline{m}=\left(m_{0}, m_{1}, \ldots, m_{S}, \ldots, m_{2^{n}-1}\right)
$$

The dimensions of the state vector $\underline{m}$ are indexed by the subsets $S \in \mathcal{S}$. The value $m_{S}$ is the number of packets which appear at every node $i \in S$ and at no node $j \in S^{C}$. Therefore, the number of packets $q(i)$ which appear at any node $i \neq s, d$ in the network is a function of $\underline{m}$. Let

$\mathcal{S}_{i}=\left\{S \in \mathcal{S} \mid\right.$ the $i^{t h}$ bit in the binary expansion of $S$ is a 1$\}$.
Then

$$
q(i)=\sum_{S \in \mathcal{S}_{i}} m_{S}, \quad q(s)=\sum_{S \in \mathcal{S}} m_{S}
$$

and the destination node $d$ retains no buffer.

\section{B. Markov Chain Evolution - Transition Model}

To understand the evolution of the Markov chain model describing the state $\underline{m}$ of the queuing system, first take an example of the network where $n=1$.

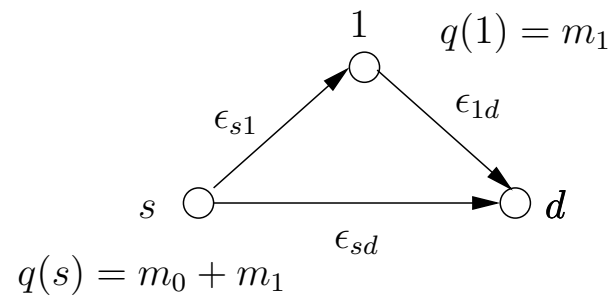

Fig. 1. A general $n=1$ wireless erasure network.

Successful transmission events can cause three different kinds of transitions to the state vector $\underline{m}$.

- There is an exogenous arrival to the system. In this case, the source node receives a new packet; the source is therefore the only node in the system which has that particular packet in its buffer. Thus, the value of $m_{0}$ is increased by 1 . This occurs at rate $\lambda$.

- A packet (which exists in some subset $S_{1}$ of nodes) can be successfully received by the destination $d$, and therefore flushed from the network. This kind of event will occur with rate

$$
\sum_{i \in S_{1}} \mu_{i d} \frac{m_{S_{1}}}{q(i)} .
$$

That is, for every node $i \in S_{1}$, we find the rate at which packets are successfully transmitted to the destination $\left(\mu_{i d}\right)$ and multiply it by the probability that the randomly selected packet was counted in the subset corresponding to $m_{S_{1}}$. Here, $m_{S_{1}}$ decreases by 1 .

- A packet (which exists in the subset $S_{1}$ ) transmitted at some node $i$ is successfully received at some subset of possible receiver nodes, at least one of which did not previously have that particular packet in its buffer. In this case, let $S_{2}$ be the new subset of nodes which have this packet. This constrains $S_{1} \subset S_{2}$, and this occurs with rate

$$
\sum_{i \in S_{1}}\left(\prod_{j \in S_{2} \backslash S_{1}} \mu_{i j} \prod_{j \notin S_{2}} \epsilon_{i j}\right) \frac{m_{S_{1}}}{q(i)} .
$$

Here, $m_{S_{1}}$ decreases by 1 (there is one less packet which is unique to the subset $S_{1}$ ) while $m_{S_{2}}$ increases by 1 . It is important to note that in this kind of transition, the subset $S_{2}$ whose variable $m_{S_{2}}$ increases must always be a superset of the subset $S_{1}$ whose variable $m_{S_{1}}$ decreases.

Each of these possible transitions and their individual rates are illustrated in Figure 2 for the $n=1$ three node network of Figure 1. 


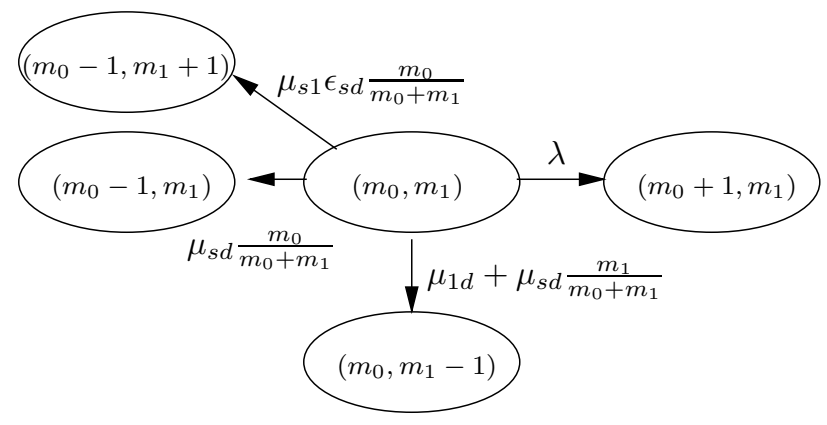

Fig. 2. Possible transitions and transition rates from a state $\left(m_{0}, m_{1}\right)$ in the $n=1$ wireless erasure network.

\section{Queue Stability and Foster's Theorem}

We desire to show that, for any arrival rate $\lambda<$ $\min _{S \in \mathcal{S}} C(S)$, all the queues in the network are stable. We refer the reader to, for example, [6] for a precise statement of the Theorem. Intuitively, Foster's Theorem states that if a Lyapunov function on the states of a Markov chain can be found, whose expected value decreases over all but a finite number of states, then the Markov chain is positive recurrent, i.e. the queues of the system are stable.

\section{Proof For the CASE $n=1$}

This section contains a demonstration of the stability proof for the simplest network, the case where $n=1$, illustrated in Figure 1. Note that for this particular network, the cut-set bound evaluates to

$$
\min \left(1-\epsilon_{s 1} \epsilon_{s d}, 1-\epsilon_{1 d}+1-\epsilon_{s d}\right)
$$

Lemma 1: The network illustrated in Figure 1 is stable for

$$
\lambda<\frac{N}{N+1} \frac{1}{1+\delta} \min \left(1-\epsilon_{s 1} \epsilon_{s d}, 1-\epsilon_{1 d}+1-\epsilon_{s d}\right)
$$

for any fixed $N>0$ and $\delta>0$.

Take $N$ to be large and $\delta$ to be small.

Proof: Consider the Lyapunov function

$$
V\left(m_{0}, m_{1}\right)=N(1+\delta)^{m_{0}}+(1+\delta)^{m_{0}+m_{1}} .
$$

This Lyapunov function is "rewarded" (i.e. decreases) when $m_{0}$ decreases and penalized when $m_{0}$ increases. When a packet is received at the relay node, the function is rewarded (while $m_{1}$ increases, $m_{0}$ simultaneously decreases) and when a packet leaves the system (i.e. $m_{1}$ decreases) the function is also rewarded.

Now, evaluate the expected change in the Lyapunov function $V\left(m_{0}, m_{1}\right)$ to determine when it is bounded away from zero:

$$
\begin{aligned}
& \lambda\left(V\left(m_{0}+1, m_{1}\right)-V\left(m_{0}, m_{1}\right)\right) \\
& \quad+\left(\mu_{1 d}+\mu_{s d} \frac{m_{1}}{m_{0}+m_{1}}\right)\left(V\left(m_{0}, m_{1}-1\right)-V\left(m_{0}, m_{1}\right)\right) \\
& \quad+\mu_{s d} \frac{m_{0}}{m_{0}+m_{1}}\left(V\left(m_{0}-1, m_{1}\right)-V\left(m_{0}, m_{1}\right)\right) \\
& \quad+\mu_{s 1} \epsilon_{s d} \frac{m_{0}}{m_{0}+m_{1}}\left(V\left(m_{0}-1, m_{1}+1\right)-V\left(m_{0}, m_{1}\right)\right)<0 .
\end{aligned}
$$

Equation (4) represents the change in Lyapunov function for all possible state transitions from the state $\underline{m}=\left(m_{0}, m_{1}\right)$, weighted by the appropriate rates to calculate the expectation. A simplification leads to

$$
\begin{aligned}
\lambda & \left(N+(1+\delta)^{m_{1}}\right) \\
& <\left(1-\epsilon_{s 1} \epsilon_{s d}\right) N \frac{m_{0}}{m_{0}+m_{1}} \frac{1}{1+\delta} \\
& +\left(\mu_{1 d}+\mu_{s d}\right)(1+\delta)^{m_{1}} \frac{1}{1+\delta} .
\end{aligned}
$$

By inspection of the first and third lines of Equation (5), note that regardless of the value of $m_{0}$, a $m_{1}^{*}$ can be found sufficiently large such that if $\lambda<\left(\mu_{s 1}+\mu_{s d}\right) \frac{1}{1+\delta}$, the inequality is fulfilled for all $m_{1}>m_{1}^{*}$. Specifically, choose $m_{1}^{*}$ such that

$$
\frac{\lambda}{\mu_{s 1}+\mu_{s d}}<\frac{(1+\delta)^{m_{1}^{*}}}{N+(1+\delta)^{m_{1}^{*}}} .
$$

Likewise, for any fixed $m_{1}<m_{1}^{*}$, choose $m_{0}>N m_{1}$, and $\lambda<\min \left(1-\epsilon_{s 1} \epsilon_{s d}, \mu_{1 d}+\mu_{s d}\right) \frac{N}{N+1} \frac{1}{1+\delta}$. Then $\lambda N$ from the first line of Equation (5) is less than the second line and $\lambda(1+$ $\delta)^{m_{1}}$ is less than the third line; the inequality is again fulfilled. Thus, there are only a finite number of states (possible only when $m_{1}<m_{1}^{*}$ and $m_{0}<N m_{1}$ ) where the expected value of Lyapunov function is increasing, and the requirements of Foster's Theorem are fulfilled.

The relevant observation is that, when $m_{1}$ dominates $m_{0}$, it is the cutset bound for the corresponding cut $S=\{s, 1\}$ which provides the critical constraint on the maximum $\lambda$ can be to keep the network stable. When $m_{0}$ dominates, the corresponding cut $S=\{s\}$ is the critical constraint on $\lambda$.

\section{Proof for General Network}

Recall Theorem 1, which we desire to prove:

Theorem 1: Under the given randomized transmission strategy, all queues in a wireless erasure network with feedback are stable as long as $\lambda<C(S)$ for all $S \in \mathcal{S}$.

For a general wireless erasure network with $n+2$ nodes, recall the Markov chain describing the system evolution described in Sections IV-A and IV-B. Foster's Theorem is utilized to demonstrate the stability of this Markov chain for a general $n+2$ node network.

In general, define the Lyapunov function $V(\underline{m})$ as

$$
V(\underline{m})=\sum_{S \in \mathcal{S}} N_{|S|}(1+\delta)^{\sum_{S^{\prime} \subseteq S} m_{S^{\prime}}}
$$

where all $N_{|S|}$ are chosen such that $N_{|S|}>N \sum_{S^{\prime} \supset S} N_{\left|S^{\prime}\right|}$ and $N>0$ and $\delta>0$ are fixed constants.

As was the case for Equation (3), the intuition for the form of this Lyapunov function is that a packet progressing though the system (transitioning from one cutset to a superset of that cutset) only decreases the value of the function. The proof of Theorem 1 follows directly from Lemma 2:

Lemma 2: The expected value of the function $V(\underline{m})$, defined in Equation (6), is increasing only on a finite number of states whenever $\lambda<\frac{N}{N+1} \frac{1}{1+\delta} \min _{S \in \mathcal{S}} C(S)$. 
Proof: First fix $S \in \mathcal{S}$, and examine the term in the main summation of Equation (6) corresponding to that $S$. Then, determine which transitions of the Markov chain effect the value of that term. Let

$$
V_{S}(\underline{m})=N_{|S|}(1+\delta)^{\sum_{S^{\prime} \subseteq S} m_{S^{\prime}}} .
$$

An arrival to the system effects every $V_{S}$, since every term $V_{S}$ contains $m_{0}$. Thus $\forall S \in \mathcal{S}$,

$$
\begin{aligned}
& V_{S}\left(m_{0}+1, m_{1}, \ldots\right)-V_{S}\left(m_{0}, m_{1}, \ldots\right) \\
& \quad=N_{|S|}(1+\delta)^{\sum_{S^{\prime} \subseteq S} m_{S^{\prime}}+1}-N_{|S|}(1+\delta)^{\sum_{S^{\prime} \subseteq S} m_{S^{\prime}}} \\
& \quad=\delta N_{|S|}(1+\delta)^{\sum_{S^{\prime} \subseteq S} m_{S^{\prime}}} .
\end{aligned}
$$

If a packet appearing in cut-set $S_{1}$ departs the system, precisely the terms $V_{S}(\underline{m})$ when $S \supseteq S_{1}$ will decrease, since they are the only terms in the Lyapunov function Equation (6) which contain $m_{S_{1}}$. For $S \supseteq S_{1}$,

$$
\begin{aligned}
& V_{S}\left(m_{0}, m_{1}, \ldots, m_{S_{1}}-1, \ldots\right)-V_{S}\left(m_{0}, m_{1}, \ldots\right) \\
& \quad=N_{|S|}(1+\delta)^{\sum_{S^{\prime} \subseteq S} m_{S^{\prime}}-1}-N_{|S|}(1+\delta)^{\sum_{S^{\prime} \subseteq S} m_{S^{\prime}}} \\
& \quad=-\delta N_{|S|}(1+\delta)^{\sum_{S^{\prime} \subseteq S} m_{S^{\prime}}-1} .
\end{aligned}
$$

The final possible transition type occurs when a packet located at the nodes in subset $S_{1}$ is successfully received at some set of nodes which did not previously have that packet, but not the destination $d$, resulting in that packet being finally in the subset $S_{2} \supset S_{1}$. Thus $m_{S_{1}}$ will decrease by 1 , and $m_{S_{2}}$ will increase by 1 . The only terms $V_{S}(\underline{m})$ that will change are those containing $m_{S_{1}}$ but not $m_{S_{2}}$. Thus, for $S$ such that $S \supseteq S_{1}$ and $S \nsupseteq S_{2}$,

$$
\begin{aligned}
& V_{S}\left(m_{0}, m_{1}, \ldots, m_{S_{1}}-1, \ldots, m_{S_{2}}+1, \ldots\right)-V_{S}\left(m_{0}, m_{1}, \ldots\right) \\
& \quad=N_{|S|}(1+\delta)^{\sum_{S^{\prime} \subseteq S} m_{S^{\prime}}-1}-N_{|S|}(1+\delta)^{\sum_{S^{\prime} \subseteq S} m_{S^{\prime}}} \\
& \quad=-\delta N_{|S|}(1+\delta)^{\sum_{S^{\prime} \subseteq S} m_{S^{\prime}}-1} .
\end{aligned}
$$

The rates for each of these three kinds of events were given in Section IV-B. To obtain the expected value of change in the Lyapunov function, sum the product of the rates of each kind of change with the value of each change. The expected increase due to arrivals should be less than the expected decrease due to departures and transitions on all but a finite number of state $\underline{m}$. The sum of changes over all of the terms must therefore satisfy

$$
\begin{aligned}
\lambda & \sum_{S \in \mathcal{S}} N_{|S|}(1+\delta)^{\sum_{S^{\prime} \subseteq S} m_{S^{\prime}}} \\
& <\sum_{S \in \mathcal{S}} \sum_{\left\{\left(S_{1}, S_{2}\right) \mid S_{1} \subset S_{2}, S_{1} \subseteq S, S_{2} \nsubseteq S\right\}} \\
& \left(\sum_{i \in S_{1}} \frac{m_{S_{1}}}{q(i)} \prod_{j \in S_{2} \backslash S_{1}} \mu_{i j} \prod_{j \notin S_{2}} \epsilon_{i j}\right) N_{|S|}(1+\delta)^{\sum_{S^{\prime} \subseteq S} m_{S^{\prime}}-1} \\
& +\sum_{S \in \mathcal{S}} \sum_{S_{1} \subseteq S}\left(\sum_{i \in S_{1}} \frac{m_{S_{1}}}{q(i)} \mu_{i d}\right) N_{|S|}(1+\delta)^{\sum_{S^{\prime} \subseteq S} m_{S^{\prime}}-1} .
\end{aligned}
$$

In the second and third lines of Equation (11), the first summation is over terms in the Lyapunov function. The second summation is over transitions of the possible pairs of $S_{1}$ and $S_{2}$ which will effect that particular term, and the third summation is over nodes which could possibly transmit and create that transition. The final terms of the third line represent the value of the change in that term $V_{S}(\underline{m})$.

Similarly, in the fourth line of Equation (11), the first summation is over the terms of the Lyapunov function, and the second is over the possible departures from the system which can effect the value of each term. Within the parentheses is the rate of those departures, and the final terms again represent the value of the change in the term $V_{S}(\underline{m})$.

We must show that Equation (11) holds for all but a finite number of states $\underline{m}$. The proof from this point further consists simply of bookkeeping, and has been omitted due to space requirements. The full proof can be found in our full paper, available on arxiv.

\section{CONCLUSION}

In this paper, we have demonstrated a parallel between the erasure channel and a network of such channels: When acknowledgment feedback is available, there exists a simple transmission strategy by which the information-theoretic capacity (calculated by the cut-set bound) can be achieved for a unicast network without any need for any coding scheme. We have described a novel randomized and decentralized strategy which requires only a surprisingly small amount of information about the network (specifically, no knowledge whatsoever about the network topology) to succeed in stabilizing the queues and achieving throughput optimality. Finally, we have proposed a non-standard, to our knowledge, exponential Lyapunov function with which to demonstrate the stability of the queuing network. The essential observation in the proof is the relationship between the definition of the state of the Markov chain (number of packets unique to each cut-set) and each of the corresponding cut-set bounds required to be fulfilled by the theorem. The problem of whether multicast capacity can be achieved without network coding in this network model remains open.

\section{REFERENCES}

[1] T. Cover and J. Thomas, Elements of Information Theory, Wiley, New York, 1991.

[2] D.S. Lun, M. Medard, and M. Effros "On coding for reliable communication over packet networks," in Proc. 42nd Annual Allerton Conference on Communication, Control, and Computing, Sept.-Oct. 2004.

[3] A. Dana, R. Gowaikar, R. Palanki, B. Hassibi, and M. Effros, "Capacity of wireless erasure networks," IEEE Trans. Inform. Theory, vol. 52, no. 3, pp. 789-804, Mar 2006.

[4] M. J. Neely and R. Urgaonkar, "Optimal Backpressure Routing in Wireless Networks with Multi-Receiver Diversity," inProc. Conference on Information Sciences and Systems, Mar. 2006.

[5] J. Schalkwijk and T. Kailath, "A coding scheme for additive noise channels with feedback-I: No bandwidth constraint," IEEE Transactions on Information Theory, vol. 12, pp. 172-182, Apr. 1966.

[6] P. Bremaud, Markov Chains, Gibbs Fields, Monte Carlo Simulation, and Queues: Springer Science, 2001. 